\title{
Estrous Cycle Monitoring in Mice with Rapid Data Visualization and Analysis
}

\author{
Leanna K. Pantier ${ }^{1, \#, ~ J i a n g ~ L i ², ~ \# ~ a n d ~ C a t h e r i n e ~ A . ~ C h r i s t i a n ~}{ }^{1,2,3, *}$
}

\begin{abstract}
1Department of Molecular and Integrative Physiology, University of Illinois at Urbana-Champaign, Urbana, IL, USA; ${ }^{2}$ Neuroscience Program, University of Illinois at Urbana-Champaign, Urbana, IL, USA; ${ }^{3}$ Beckman Institute for Advanced Science and Technology, University of Illinois at Urbana-Champaign, Urbana, IL, USA

*For correspondence: cathchri@illinois.edu

\#Contributed equally to this work
\end{abstract}

[Abstract] The estrous cycle provides a readout of reproductive health in female laboratory rodents, and estrous cycle stage can be an important physiological variable. Accurate assessment of estrous cycle stage is also important in producing timed pregnancies for developmental studies. Here, we provide a protocol for evaluation of estrous cycle stage through a minimally invasive procedure of acquiring cells lining the vaginal cavity and immediate microscopic visual assessment of these cells without drying or staining. When performed over several consecutive days, the pattern of progression through the four main stages of the estrous cycle, and disruptions to this pattern, can be determined. We also present software that enables more efficient cycle stage data analysis and pattern visualization. These protocols and tools will thus facilitate the incorporation of female animals in laboratory experiments and enhance the assessment of relationships between the reproductive cycle and overall physiology and behavior.

Keywords: Mouse, Reproduction, Fertility, Reproductive cycle, Behavior, Physiology, Endocrinology, Rodent

[Background] The reproductive health of an animal is an important parameter of overall health and physiological function. In female laboratory rodents, assessment of estrous cycle stage over several consecutive days allows for short- and long-term tracking of each animal's cyclicity, and thus reproductive health. In mice and rats, there are typically four stages that comprise the estrous cycle (in order): proestrus; estrus; metestrus; and diestrus, with diestrus often spanning two days (diestrus I and diestrus II). A healthy animal will move through the stages successively across 4-5 days on average (Byers et al., 2012; Cora et al., 2015).

Proper assessment of estrous cycle stage is important in producing timed pregnancies for developmental studies, as female mice are most receptive to mating when introduced to a male on proestrus or estrus (Mader et al., 2009; Smarr et al., 2016). Furthermore, certain animal behavior, physiology, and pharmacology parameters can change with estrous cycle stage in rodents (Maguire et al., 2005; Cordeau et al., 2008; Milad et al., 2009; Lebron-Milad et al., 2013; Cushman et al., 2014; McHenry et al., 2017; Yagi et al., 2017; Broestl et al., 2018; Cordeira et al., 2018; Hirsch et al., 2018; Kaur et al., 2018; Santos et al., 2018; Johnson et al., 2019). Impaired mouse estrous cyclicity can 
provide a readout of compromised reproductive function, for example in toxicological studies (Hannon et al., 2014), models of endocrine disorders (Sullivan and Moenter, 2004; Novaira et al., 2014; Babwah et al., 2015), and in models of neurological disorders (Fawley et al., 2012; Jaini et al., 2015; Li et al., 2017; Li et al., 2018). Changes in vaginal wall electrical impedance in rats and mice can be measured with specialized monitors, but these changes may lack accuracy and may only be detected on certain cycle stages (Singletary et al., 2005). Similarly, changes in the vaginal opening can be determined by visual observation, but again only on certain stages, such as proestrus and estrus (Byers et al., 2012). By contrast, estrous cycle stage can be readily assessed in a minimally invasive manner through vaginal cytology, as has been documented for a century (Allen, 1922). Although these samples may be dried and stained for preservation and examination, such as with Giemsa or Toluidine blue stains (Cora et al., 2015), this process is time-consuming and may not be amenable to continual estrous cycle monitoring in large numbers of animals. With proper training and practice, however, it is possible to determine cycle stage based on direct examination of cell morphology and number without additional staining or cell preservation procedures. Here, we provide a protocol for this estrous cycling process for mice.

Funding agencies, including the U.S. National Institutes of Health and the Canadian Institutes of Health Research, have recently implemented policies requiring researchers to address sex as a biological variable, necessitating the increased inclusion of female animals in biomedical research (Clayton and Collins, 2014; Clayton, 2018; Canadian Institutes of Health Research, 2019). With greater use of female animals in rodent studies, and larger numbers of animals that may require estrous cycle monitoring, there is an added need for tools that expedite the processes of estrous cycle data analysis. To this end, we also present a novel software program that facilitates a greater throughput of mouse estrous cycle data acquisition, analysis, and visualization, which can also be used for other common laboratory rodents, such as rats. This tool allows for improved understanding of patterns or changes that are occurring in each animal across large cohorts.

\section{Materials and Reagents}

1. Sterile $200 \mu$ l pipette tips (Fisher Scientific, Fisherbrand Redi-Tip 1-200 $\mu$ l pipette tips, catalog number: 02-681-147)

2. Nitrile gloves (VWR, WWR Powder-Free Nitrile Examination Gloves, catalog number: 82026)

3. $50 \mathrm{ml}$ Falcon centrifuge tube (Fisher Scientific, catalog number: 14-432-22)

4. Plain microscope slides (Denville Scientific Inc., Denville Ultra Plain microscope slides, catalog number: M1001)

5. Microscope slide cover slips (Denville Scientific Inc., Denville Ultra Microscope Cover Glass, catalog number: M1100-02)

6. Post-pubertal and sexually mature ( $>42$ days old) female mice (any strain)

7. Sterile 10x PBS (Fisher Scientific, Lonza BioWhittaker Phosphate Buffered Saline (10x), catalog number: 11669980) 
8. Deionized $\mathrm{H}_{2} \mathrm{O}$ (MilliporeSigma Synergy Ultrapure Water Purification System, manufacturer number: SYNSOHFUS)

\section{Equipment}

1. Compound binocular microscope with $4 x$ and 10x magnification objectives (Olympus CX43 Biological Microscope)

2. 20-200 $\mu$ l pipette (Gilson, Pipetman P200L, 20-200 $\mu$ capacity, catalog number: FA10005M)

3. Tube rack (Fisherbrand 4-way Tube Rack, catalog number: 03-448)

\section{Software}

1. Microsoft Excel

2. Python (www.python.org)

3. OriginPro (or similar statistical software)

Note: Files for Python functions to improve the speed of mouse estrous cycle graph visualization are available at https://github.com/ChristianLabUIUC/EstrousCycle. The procedure for implementing and using these files is described below.

\section{Procedure}

Note: These procedures should be performed at the same time each day. For example, our lab times these procedures to Zeitgeber time (ZT) 1-5, relative to lights-off at ZT 12.

A. Collection and classification of mouse vaginal smear samples

1. In a $50 \mathrm{ml}$ Falcon tube, dilute $10 x$ PBS to a $1 x$ PBS solution by adding $5 \mathrm{ml}$ of $10 x$ PBS to $45 \mathrm{ml}$ of distilled water and shaking gently to mix. Place tube in tube rack and prepare a clean slide for the sample(s).

2. Using a $20-200 \mu l$ pipette, aspirate $20 \mu \mathrm{l}$ of $1 \mathrm{x}$ PBS into a $200 \mu \mathrm{l}$ pipette tip.

3. Retrieve mouse from cage, and place on cage top while gently holding the tail for restraint. Allow the mouse to grip the top of the cage lid. Orient the mouse so that its posterior is visible.

4. Using the non-dominant hand, gently hold the tail of the mouse between the thumb and forefinger and gently pull upwards so that back feet come off the cage top. Use the ring and pinky fingers to gently push on the mouse's hind quarters to angle the hips upward so as to easily view and access the vagina (Figure 1). 


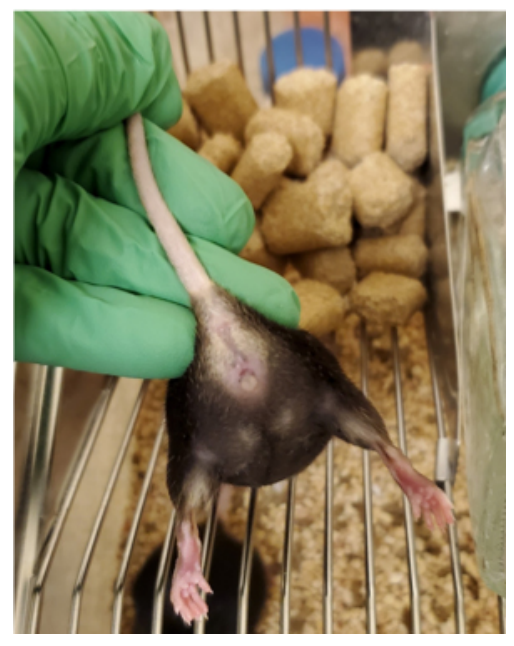

Figure 1. Orientation of mouse for sample collection

5. With the dominant hand, pick up the pipette. Insert the pipette tip into the mouse's vagina approximately $0.2 \mathrm{~cm}$ deep, making sure to not insert the tip too far and cause internal injury or stress (Figure 2).

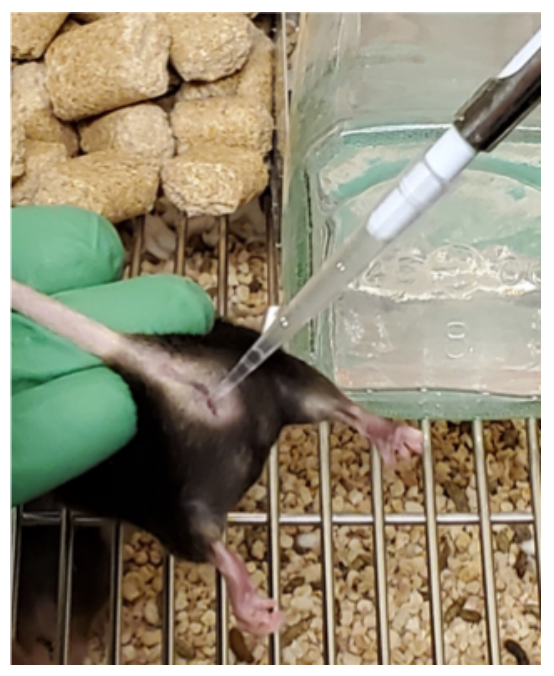

Figure 2. Gentle insertion of pipette tip into vaginal cavity

6. Pipette the solution in and out 1-3 times, and aspirate as much liquid as possible to use for the smear sample.

7. Replace the mouse in its home cage.

8. Gently expel the smear sample onto the slide, making a vertical ellipse shape (Figure 3). 


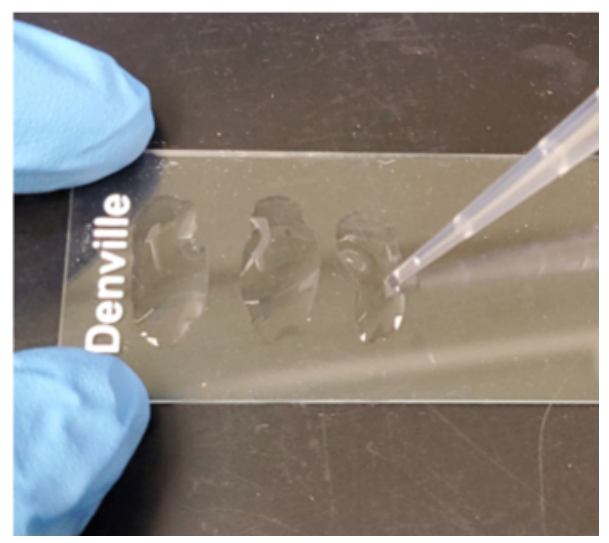

Figure 3. Extraction of smear sample onto microscope slide. Note that multiple smears can be placed on the same slide.

9. Repeat this step for subsequent mice until there is no room left on the slide for samples (usually 5 to 8 samples per slide).

10. To evaluate the samples, place slide on the stage of a compound binocular microscope. Identify the stage for each sample, and record the information in a lab notebook.

11. Once vaginal smears have been collected on a slide, they must be analyzed and classified into stages based on the cell type(s) present in each individual smear. There are four canonical cycle stages: proestrus (nucleated epithelial cells), estrus (cornified epithelial cells), metestrus (approximately 50\% cornified epithelial cells and leukocytes), and diestrus (leukocytes at very high density). A stage in which only a few cells are obtained, and in which all the cells are leukocytes, can be observed, particularly when diestrus is prolonged. For the purposes of characterizing these smears and entering the data into the analysis program, give these smears the name "few". Only one stage can be entered into the analysis program; if a smear appears to have more than one cell type (e.g., both nucleated and cornified epithelial cells), record the stage that the majority cell type represents (Figure 4).
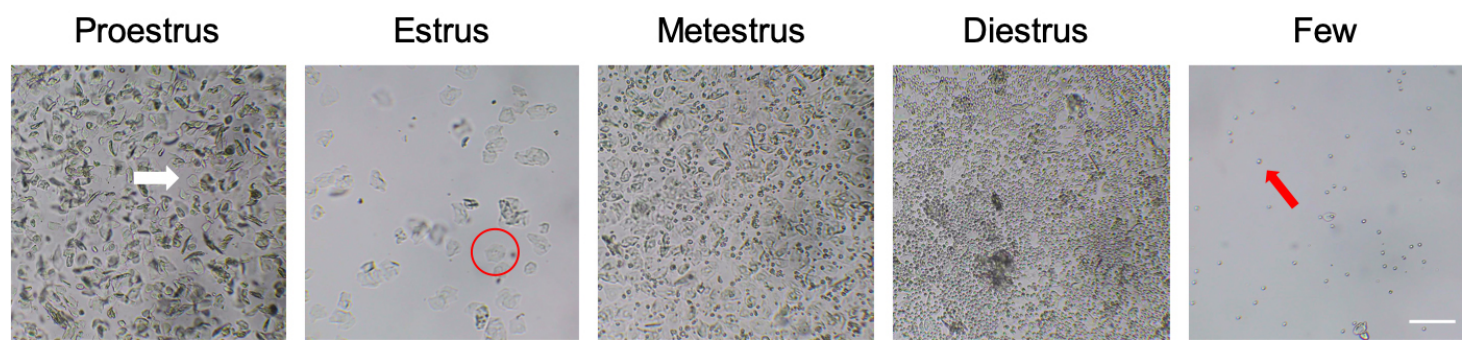

Figure 4. Representative smears illustrating cytology for each cycle stage. The white arrow in the "Proestrus" example indicates a nucleated epithelial cell. The red circle in the "Estrus" example indicates a cornified epithelial cell. The red arrow in the "Few" example indicates a leukocyte. Images obtained at 10x magnification. Scale bar $=100 \mu \mathrm{m}$.

\section{B. Application of Python code for data visualization and analysis}


1. Generate an Excel file that contains the following columns:

a. Mouse id: a unique identifier for each mouse.

b. CycleDate: the date on which the estrous cycle monitoring was performed.

c. CycleStage: the estrous cycle stage. The function will only take P, E, M, D, and FEW as valid estrous cycle stage data terms. The first four terms represent proestrus, estrus, metestrus, and diestrus, respectively. See Note 7 for full descriptions and visualizations of each stage. "FEW" is described as the stage following diestrus, when leukocytes are clearly still visible in the smear, but are significantly diminished in number compared to a full diestrus smear. Days listed as FEW will be plotted as $D$ for the purposes of data visualization. Invalid data will be discarded.

d. Time: gives a label to the period of estrous cycle monitoring. For example, if estrous cycle monitoring is performed on the same mouse before and after a drug treatment, label the data as 1 for the time before the treatment and label the data as 2 for the time after the treatment. This function enables efficient visualization and plotting of the different time periods.

Note: Each row will represent the smear for a given mouse on a single day. Multiple mice can be included in the same spreadsheet, as long as they are labeled with distinct IDs. Ensure that the input Excel file has columns named exactly as described; otherwise, the plot will not be properly generated.

2. Use the functions implemented in the pyEstrousCycle.py file.

a. plotCycles(fileDir, animallD, saveOutPutFile, resolution, time, colors)

Note: This function will generate the estrous cycle plot for a given mouse (example in Figure 5).

- fileDir: the directory for the input file

- animallD: a unique identifier for each mouse

- saveOutPutFile: set to True to save the plot to local a directory. The default is False.

- Resolution: set the dpi value for the plot. The default is 80 .

- Time: if needed, select time periods to group data into different sessions of monitoring in a list of values, e.g., [1, 2] to indicate session 1 and session 2, respectively

- Colors: select colors for different time periods, listed in the order of assignment to each period of monitoring; e.g., ['black', 'red'] to assign black to session 1 and red to session 2.

b. plotAllofThem(fileDir, saveOutPutFile, resolution, time, colors)

Note: This function will generate estrous cycle plots for all mice in the input file. Input argument settings are the same as in the plotCycles function.

c. percentage_table = analysis(fileDir, saveOutPutFile)

Note: This function will calculate the percentage of time spent in each cycle stage for each mouse. Set saveOutPutFile to True to save the result table as an Excel file in the local directory. 
3. Example code, estrous cycle data, and an example output file are provided on Github at the link above. Run the example.py code to learn how to use the Python functions and to generate example estrous cycle plots.

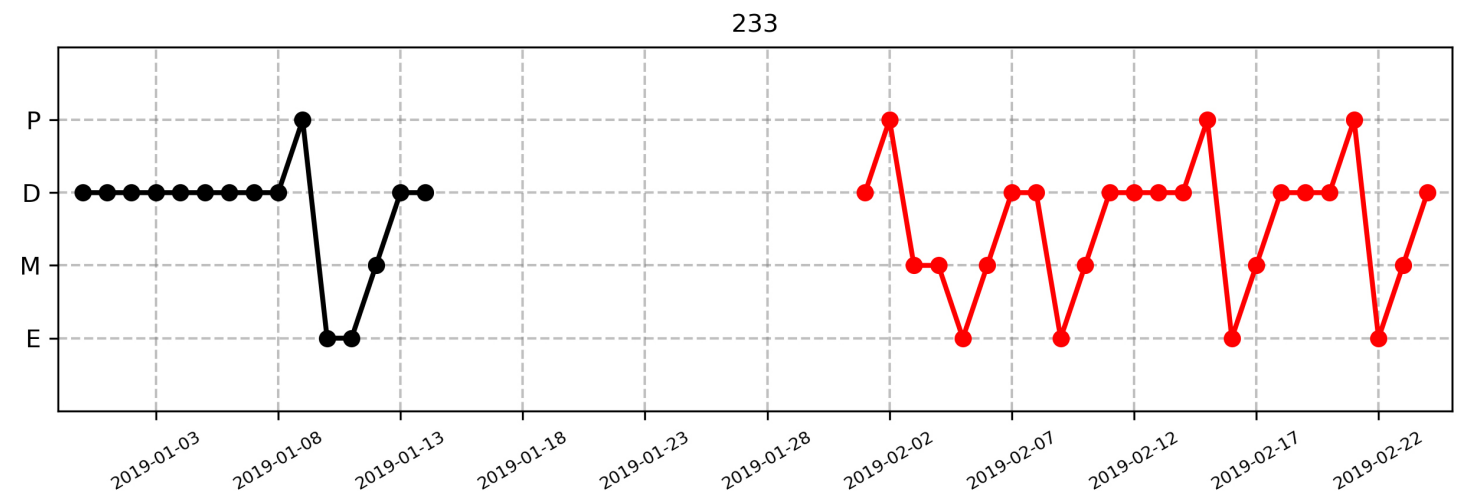

Figure 5. Example estrous cycle plot for an individual mouse generated from the function

plotCycles. The black circles and lines indicate cycle stage assessment during the first session of monitoring, and the red circles and lines indicate assessment during a second time. The dates of assessment are on the $\mathrm{X}$-axis, and the observed cycle stage is on the $\mathrm{Y}$-axis. $\mathrm{P}=$ proestrus; $D=$ diestrus; $M=$ metestrus; $E$ = estrus. The number at the top of the graph signifies the mouse ID number. Note that these data were obtained from an epileptic mouse, and that during the first period of monitoring, this mouse was placed in a new environment for continuous electroencephalogram (EEG) recording, thus giving rise to more variation and irregularity in the estrous cycle pattern. Nevertheless, this example illustrates that the data acquisition and plotting method presented here can accurately reflect both regular and disrupted cycles.

\section{Data analysis}

1. Estrous cycle length

Manually calculate the estrous cycle length for a given mouse from the estrous cycle plot. A complete estrous cycle is defined as the days between 2 days of estrus, with metestrus, diestrus and proestrus occurring in between (also, see legend for Figure 6 below).

An example illustrating quantification of estrous cycle length for a mouse is provided in Figure 6. There are eight estrous cycles identified in this plot. The cycle length for each cycle is given in Table 1. Note that cycles 1 and 8 are not complete and thus the cycle length is indicated as the minimum value possible, based on the collected data from available days covering the period of monitoring. Pool the data for all cycles for a given mouse to calculate that mouse's average cycle length. Once the average cycle length has been calculated for each mouse, apply appropriate statistical tests in OriginPro or other statistical software to compare cycle length between treatment groups. 


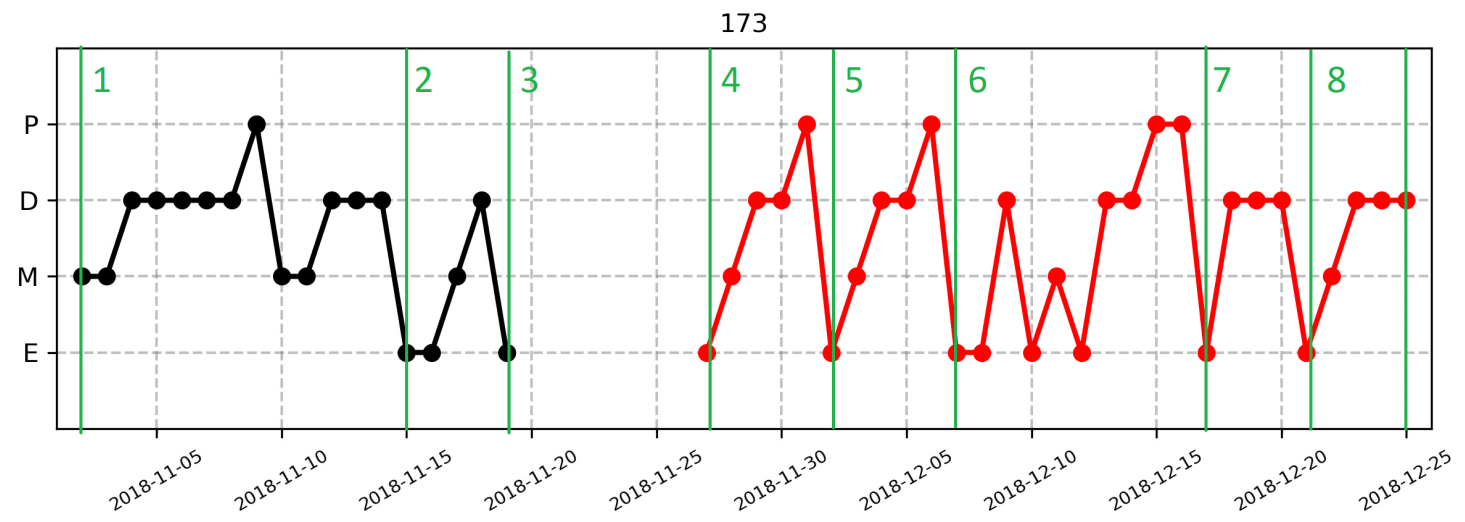

Figure 6. Designation of individual estrous cycles. In this example plot, two sessions of monitoring on either side of a 5-day break are depicted. Green lines and numbers indicate the start of each cycle. Note that frequently, the standard proestrus and metestrus cycle stages are too brief to be completely captured. If a proestrus or a metestrus day is missing during an estrous cycle, refer to the estrous cycle experiment notes to estimate if it did occur. For example, cycle 7 in this figure does not show full proestrus or metestrus, but if the experiment notes state that there were days with smears indicating a transition from estrus to metestrus, as well as a transition from proestrus to estrus, cycle 7 could be identified as a complete cycle. Also note that the green line at the end of cycle 8 is automatically generated to mark the last day of monitoring. However, because cycle 8 was not complete before the end of monitoring, it would not be included in analyses of cycle length. Note also that, as in Figure 5, these data are from an epileptic mouse initially exposed to EEG recording during the first session of monitoring, and thus both disrupted (e.g., cycles 1, 2, 6, and 7) and regular cycles (e.g., cycles 4 and 5) are obtained, but that the algorithm can illustrate the cycle lengths for both of these patterns.

Table 1. Length of estrous cycles indicated in the plot in Figure 6. Note that cycle 3 represents a gap in monitoring. This 5 -day span both begins and ends with estrus, suggesting a complete cycle likely occurred. However, because of the lack of data for these days, this cycle would not be included in data analyses (denoted in the table with an asterisk).

\begin{tabular}{ll}
\hline Cycle & Length (days) \\
\hline 1st & $>13$ \\
2nd & 4 \\
3rd & $5^{*}$ \\
4th & 5 \\
5th & 5 \\
6th & 10 \\
7th & 4 \\
8th & $>5$ \\
\hline
\end{tabular}

2. Percentage of time spent in each cycle stage 
The data for the percentage of time that a mouse spends in each cycle stage will be generated by the percentage_table function. (Note that the percentage is given in the generated spreadsheet as a value between 0 and 1 ; multiply by 100 to get the final percentage value.) Pool data from all mice for a given experimental group or session of monitoring and apply appropriate statistical tests in OriginPro or other statistical software.

\section{$\underline{\text { Notes }}$}

1. Do not use dipped or charged microscope slides. Slides may be washed in labware detergent and reused as needed.

2. During diestrus stages, it can be common for vaginal mucus to block the pipette tip. In order to collect a good-sized sample, gently move or rotate the tip in the vagina to get proper aspiration suction. It may also be helpful to aspirate another $20 \mu \mathrm{l}$ of $1 \mathrm{x}$ PBS to bolster the amount of liquid available.

3. When first learning and practicing the procedure of smear acquisition and assessment, it is best to keep the number of smears per slide low to prevent the samples from drying out as successive samples are obtained, thus compromising accurate analysis.

4. For storage of visual smear data for later assessment, one can use a microscope-mounted digital camera connected to a computer and save images to designated folders containing smear images for a given mouse for each day of cycling.

5. Excessive manual stimulation of the vaginal cavity can potentially induce pseudopregnancy in rodents (Adler and Zoloth, 1970; Yang et al., 2009). Stress can also negatively affect reproductive functioning, the neural control of reproduction, and the estrous cycle in rodents (Breen et al., 2012; Acevedo-Rodriguez et al., 2018). Therefore, it is imperative to be as gentle as possible in inserting the pipette tip and expelling/retracting PBS liquid into and from the vaginal cavity. Some mice may require habituation to handling and the cell collection procedure before regular cycles can be obtained. Our laboratory's policy is that an adult female mouse that does not show a regular cycle within 3 weeks of monitoring is excluded from further experimentation and study.

6. If a microscope is not present in the vicinity of the area where smears are collected, small circular or square cover slips can be placed on each smear to stabilize the sample for transport. Ensure that smears are placed at sufficient distance on the slide so that they do not mix when under a coverslip.

7. Although the implementation of the Python code presented here can provide for more rapid data visualization and analysis, it should be noted that generation of cycle plots and quantification of cycle length and percentage of time spent in each cycle stage can also be performed manually in Excel or another similar spreadsheet program by converting each cycle stage designation to a number (e.g., estrus $=1$, metestrus $=2$, and so on), and then plotting these numbers as a function of the day of monitoring. 


\section{Acknowledgments}

This work was supported by National Institutes of Health/National Institute of Neurological Disorders and Stroke (NIH/NINDS) grants R01 NS105825 and R03 NS103029 (C.A.C.). This protocol is adapted from Li et al., 2017 and 2018.

\section{Competing interests}

The authors declare no competing interests.

\section{Ethics}

All animal procedures were approved by the Institutional Animal Care and Use Committee of the University of Illinois at Urbana-Champaign (protocol number 17183).

\section{References}

1. Acevedo-Rodriguez, A., Kauffman, A. S., Cherrington, B. D., Borges, C. S., Roepke, T. A. and Laconi, M. (2018). Emerging insights into hypothalamic-pituitary-gonadal axis regulation and interaction with stress signalling. $J$ Neuroendocrinol 30(10): e12590.

2. Adler, N. T. and Zoloth, S. R. (1970). Copulatory behavior can inhibit pregnancy in female rats. Science 168(3938): 1480-1482.

3. Allen, E. (1922). The oestrous cycle in the mouse. Am J Anat 30(3): 297-371.

4. Babwah, A. V., Navarro, V. M., Ahow, M., Pampillo, M., Nash, C., Fayazi, M., Calder, M., Elbert, A., Urbanski, H. F., Wettschureck, N., Offermanns, S., Carroll, R. S., Bhattacharya, M., Tobet, S. A. and Kaiser, U. B. (2015). GnRH neuron-specific ablation of $\mathrm{Ga}_{\mathrm{q} / 11}$ results in only partial inactivation of the neuroendocrine-reproductive axis in both male and female mice: In vivo

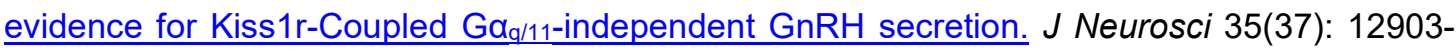
12916.

5. Breen, K. M., Thackray, V. G., Hsu, T., Mak-McCully, R. A., Coss, D. and Mellon, P. L. (2012). Stress levels of glucocorticoids inhibit LHbeta-subunit gene expression in gonadotrope cells. Mol Endocrinol 26(10): 1716-1731.

6. Broestl, L., Worden, K., Moreno, A. J., Davis, E. J., Wang, D., Garay, B., Singh, T., Verret, L., Palop, J. J. and Dubal, D. B. (2018). Ovarian cycle stages modulate Alzheimer-Related cognitive and brain network alterations in female mice. eNeuro 5(6). DOI: 10.1523/ENEURO.0132-17.2018.

7. Byers, S. L., Wiles, M. V., Dunn, S. L. and Taft, R. A. (2012). Mouse estrous cycle identification tool and images. PLoS One 7(4): e35538.

8. Clayton, J. A. (2018). Applying the new SABV (sex as a biological variable) policy to research 
and clinical care. Physiol Behav 187: 2-5.

9. Clayton, J. A. and Collins, F. S. (2014). Policy: NIH to balance sex in cell and animal studies. Nature 509(7500): 282-283.

10. Cora, M. C., Kooistra, L. and Travlos, G. (2015). Vaginal cytology of the laboratory rat and mouse: Review and criteria for the staging of the estrous cycle using stained vaginal smears. Toxicol Pathol 43(6): 776-793.

11. Cordeau, P., Jr., Lalancette-Hebert, M., Weng, Y. C. and Kriz, J. (2008). Live imaging of neuroinflammation reveals sex and estrogen effects on astrocyte response to ischemic injury. Stroke 39(3): 935-942.

12. Cordeira, J., Kolluru, S. S., Rosenblatt, H., Kry, J., Strecker, R. E. and McCarley, R. W. (2018). Learning and memory are impaired in the object recognition task during metestrus/diestrus and after sleep deprivation. Behav Brain Res 339: 124-129.

13. Cushman, J. D., Moore, M. D., Olsen, R. W. and Fanselow, M. S. (2014). The role of the $\delta$ GABA(A) receptor in ovarian cycle-linked changes in hippocampus-dependent learning and memory. Neurochem Res 39(6): 1140-1146.

14. Fawley, J. A., Pouliot, W. A. and Dudek, F. E. (2012). Pilocarpine-induced status epilepticus and subsequent spontaneous seizures: lack of effect on the number of gonadotropin-releasing hormone-positive neurons in a mouse model of temporal lobe epilepsy. Neuroscience 203: 153159.

15. Hannon, P. R., Peretz, J. and Flaws, J. A. (2014). Daily exposure to Di(2-ethylhexyl) phthalate alters estrous cyclicity and accelerates primordial follicle recruitment potentially via dysregulation of the phosphatidylinositol 3-kinase signaling pathway in adult mice. Biol Reprod 90(6): 136.

16. Hirsch, M. M., Brusco, J., Vaccaro, T., Margis, R., Moreira, J. E., Gottfried, C. and Rasia-Filho, A. A. (2018). Sex differences and estrous cycle changes in synaptic plasticity-related microRNA in the rat medial amygdala. Neuroscience 379: 405-414.

17. Jaini, R., Altuntas, C. Z., Loya, M. G. and Tuohy, V. K. (2015). Disruption of estrous cycle homeostasis in mice with experimental autoimmune encephalomyelitis. $J$ Neuroimmunol 279: 71-74.

18. Johnson, A. R., Thibeault, K. C., Lopez, A. J., Peck, E. G., Sands, L. P., Sanders, C. M., Kutlu, M. G. and Calipari, E. S. (2019). Cues play a critical role in estrous cycle-dependent enhancement of cocaine reinforcement. Neuropsychopharmacology 44(7): 1189-1197.

19. Kaur, S., Benton, W. L., Tongkhuya, S. A., Lopez, C. M. C., Uphouse, L. and Averitt, D. L. (2018). Sex differences and estrous cycle effects of peripheral serotonin-evoked rodent pain behaviors. Neuroscience 384: 87-100.

20. Lebron-Milad, K., Tsareva, A., Ahmed, N. and Milad, M. R. (2013). Sex differences and estrous cycle in female rats interact with the effects of fluoxetine treatment on fear extinction. Behav Brain Res 253: 217-222.

21. Li, J., Kim, J. S., Abejuela, V. A., Lamano, J. B., Klein, N. J. and Christian, C. A. (2017). 
Please cite this article as: Pantier et. al., (2019). Estrous Cycle Monitoring in Mice with Rapid Data Visualization and Analysis,Bio-protocol 9 (17): e3354.

Disrupted female estrous cyclicity in the intrahippocampal kainic acid mouse model of temporal lobe epilepsy. Epilepsia Open 2(1): 39-47.

22. Li, J., Robare, J. A., Gao, L., Ghane, M. A., Flaws, J. A., Nelson, M. E. and Christian, C. A. (2018). Dynamic and sex-specific changes in gonadotropin-releasing hormone neuron activity and excitability in a mouse model of temporal lobe epilepsy. eNeuro 5(5): e0273.

23. Mader, S. L., Libal, N. L., Pritchett-Corning, K., Yang, R. and Murphy, S. J. (2009). Refining timed pregnancies in two strains of genetically engineered mice. Lab Anim (NY) 38(9): 305-310.

24. Maguire, J. L., Stell, B. M., Rafizadeh, M. and Mody, I. (2005). Ovarian cycle-linked changes in GABA(A) receptors mediating tonic inhibition alter seizure susceptibility and anxiety. Nat Neurosci 8(6): 797-804.

25. McHenry, J. A., Otis, J. M., Rossi, M. A., Robinson, J. E., Kosyk, O., Miller, N. W., McElligott, Z. A., Budygin, E. A., Rubinow, D. R. and Stuber, G. D. (2017). Hormonal gain control of a medial preoptic area social reward circuit. Nat Neurosci 20(3): 449-458.

26. Milad, M. R., Igoe, S. A., Lebron-Milad, K. and Novales, J. E. (2009). Estrous cycle phase and gonadal hormones influence conditioned fear extinction. Neuroscience 164(3): 887-895.

27. Novaira, H. J., Sonko, M. L., Hoffman, G., Koo, Y., Ko, C., Wolfe, A. and Radovick, S. (2014). Disrupted kisspeptin signaling in GnRH neurons leads to hypogonadotrophic hypogonadism. Mol Endocrinol 28(2): 225-238.

28. Canadian Institutes of Health Research. (2019). How to integrate sex and gender into research. http://www.cihr-irsc.gc.ca/e/50836.html.

29. Santos, V. R., Kobayashi, I., Hammack, R., Danko, G. and Forcelli, P. A. (2018). Impact of strain, sex, and estrous cycle on gamma butyrolactone-evoked absence seizures in rats. Epilepsy Res 147: $62-70$.

30. Singletary, S. J., Kirsch, A. J., Watson, J., Karim, B. O., Huso, D. L., Hurn, P. D. and Murphy, S. J. (2005). Lack of correlation of vaginal impedance measurements with hormone levels in the rat. Contemp Top Lab Anim Sci 44(6): 37-42.

31. Smarr, B. L., Zucker, I. and Kriegsfeld, L. J. (2016). Detection of successful and unsuccessful pregnancies in mice within hours of pairing through frequency analysis of high temporal resolution core body temperature data. PLoS One 11(7): e0160127.

32. Sullivan, S. D. and Moenter, S. M. (2004). Prenatal androgens alter GABAergic drive to gonadotropin-releasing hormone neurons: implications for a common fertility disorder. Proc Natl Acad Sci U S A 101(18): 7129-7134.

33. Yagi, S., Drewczynski, D., Wainwright, S. R., Barha, C. K., Hershorn, O. and Galea, L. A. M. (2017). Sex and estrous cycle differences in immediate early gene activation in the hippocampus and the dorsal striatum after the cue competition task. Horm Behav 87: 69-79.

34. Yang, J. J., Larsen, C. M., Grattan, D. R. and Erskine, M. S. (2009). Mating-induced neuroendocrine responses during pseudopregnancy in the female mouse. $J$ Neuroendocrinol 21(1): 30-39. 